\title{
Muddy clinical waters: a missed betel nut in the bronchus
}

\author{
Sunil Karande, ${ }^{1}$ Pradeep Vaideeswar, ${ }^{2}$ Mamta Muranjan ${ }^{1}$
}

${ }^{1}$ Department of Pediatrics, Seth GS Medical College \& KEM

Hospital, Mumbai,

Maharashtra, India

${ }^{2}$ Department of Pathology (Cardiovascular \& Thoracic Division), Seth GS Medical College \& KEM Hospital, Mumbai, Maharashtra, India

\section{Correspondence to} Professor Sunil Karande, karandesunil@yahoo.com

Accepted 4 November 2015

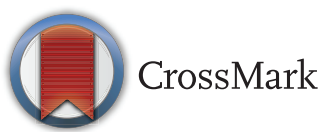

To cite: Karande $S$, Vaideeswar P, Muranjan M. BMJ Case Rep Published online: [please include Day Month Year] doi:10.1136/ bcr-2015-212919

\section{SUMMARY}

A toddler presented with a 5-month history of recurrent episodes of cough, wheezing and fever. Before referral, the toddler had been initially diagnosed as having bronchial asthma and later as having pulmonary tuberculosis. On examination, the patient was febrile and had severe respiratory distress. Chest radiograph and high-resolution CT of the chest revealed collapse of the entire left lung with diffuse bronchiectasis along with a grossly hyperinflated right lung. CT virtual bronchoscopy did not reveal any foreign body. The parents denied any history suggestive of foreign body aspiration and refused consent for rigid bronchoscopy. Nine days after admission, chest physiotherapy was inadvertently prescribed to the patient. Within an hour, the patient experienced acute respiratory deterioration and died. Autopsy revealed a piece of betel nut in the right main bronchus; it had got dislodged from its initial site in the left main bronchus following the chest physiotherapy session.

\section{BACKGROUND}

Bronchial foreign body (FB) aspiration is a common cause of respiratory compromise in children aged $1-3$ years of age. ${ }^{1-3}$ Delayed diagnosis is not uncommon as parents often do not witness the aspiration episode ('acute choking/coughing'). ${ }^{1-3}$ Persistent cough, recurrent wheezing/pneumonia and fever are the most common presenting clinical features in young children with delayed diagnosis of bronchial FB aspiration. ${ }^{1-3}$ Unilateral diminished breath sounds with contralateral overdistension on the chest radiograph are the most predictive features for the potential diagnosis of chronic bronchial FB aspiration. ${ }^{1-3}$ An undiagnosed, retained bronchial FB is known to cause serious complications such as atelectasis, bronchiectasis and bronchial fistula, and it can even get secondarily dislodged and cause acute respiratory deterioration. ${ }^{1-3}$

We report a case of a toddler in whom the diagnosis of a chronic bronchial FB was missed, with tragic consequences. This occurred in spite of taking a detailed clinical history, performing a thorough clinical examination and carrying out relevant radiological investigations. We wish to share the lesson that we learnt from this inadvertent error.

\section{CASE PRESENTATION}

A toddler was admitted to our hospital, with recurrent episodes of cough, wheezing and fever for the past 5 months. A local general practitioner had made a diagnosis of bronchial asthma and treated these episodes with oral antibiotics and nebulisation with ipratropium bromide and budesonide. The toddler would get temporarily relieved, but the same symptoms would recur after a period of 2-3 weeks. Initially, the cough was dry; but over the next 2-3 months it became a phlegmy-sounding 'wet' cough. One month prior to admission at our hospital, the parents had consulted a private paediatrician, who made a diagnosis of pulmonary tuberculosis ('which was stated on the prescription'); and started the toddler on standard four-drug antituberculosis treatment (isoniazid+rifampicin + ethambutol+pyrazinamide). We tried to reach the private paediatrician but details of the earlier investigations remained unavailable.

Five days prior to admission to our hospital, the toddler had again developed cough, wheezing and fever. This time, since there was no response to the usual treatment with oral antibiotics and nebulisation, the toddler was referred for further management. On examination, the patient was febrile, with a heart rate of $130 \mathrm{bpm}$ and respiratory rate of $58 / \mathrm{min}$ associated with bilateral intercostal, subcostal and suprasternal retractions indicating severe respiratory distress. Blood pressure was normal; 96/ $70 \mathrm{~mm} \mathrm{Hg}$. Pulse oximetry revealed $\mathrm{SpO}_{2}$ of $88 \%$ on room air, which normalised to $97 \%$ on nasal oxygen $(6 \mathrm{~L} / \mathrm{min})$. The toddler's weight was $10 \mathrm{~kg}$ and height was $94 \mathrm{~cm}$. The patient's weight-for-height was below $-3 \mathrm{SD}$ of the WHO standards indicating severe acute malnutrition (SAM). ${ }^{4}$ Pallor was present, but there was no cyanosis or clubbing. Respiratory system examination revealed tracheal deviation to the left and crowding of the ribs in the left inframammary and axillary regions. On percussion, there was a hyper-resonant note all over the right side and dull note all over the left side of the chest. On auscultation, bilateral wheeze and scattered coarse crackles on the left side were heard. Air entry was decreased bilaterally but compared to the right side it was markedly decreased all over the left side of the chest. Other system examinations were normal.

On detailed enquiry, the parents denied any history suggestive of FB aspiration. There was no history of stridor, no contact with tuberculosis and the patient had not suffered from measles or pertussis in the past. There was no personal history of atopic dermatitis or allergic rhinitis or family history of bronchial asthma. The patient's immunisation status was up-to-date. Our initial clinical diagnosis was collapse of the left lung with bronchiectasis and compensatory emphysema of the right lung with superadded lower respiratory tract infection. 


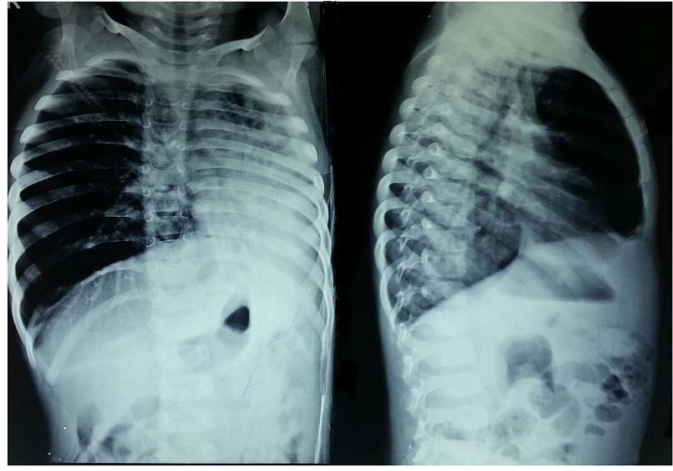

Figure 1 Chest radiograph (posterioanterior and left-lateral views) showing collapse of the left lung with bronchiectatic changes and massive compensatory hyperinflation of the right lung with mediastinal shift to the left.

\section{INVESTIGATIONS}

Blood investigations on admission showed a haemoglobin level of $92 \mathrm{~g} / \mathrm{L}$ and leucocytosis (white cell count of $21.3 \times 10^{9} / \mathrm{L}$, (92\% neutrophils, 8\% lymphocytes) and platelet count of $\left.420 \times 10^{9} / \mathrm{L}\right)$. Peripheral blood smear examination for malarial parasite was negative. Random blood sugar at time of admission was $4.9 \mathrm{mmol} / \mathrm{L}$. Blood cultures were sterile. Mantoux test was negative. Gastric lavages for acid-fast bacilli were negative. Chest radiograph revealed collapse of the left lung with bronchiectatic changes, with massive compensatory hyperinflation of the right lung (figure 1). On second day of admission, a highresolution CT (HRCT) of the chest was performed, which revealed collapse of all segments of the left lung, with cystic bronchiectatic changes and massive compensatory hyperinflation of the right lung resulting in its herniation to the left, and consequent mediastinal shift to the left (figure 2). An urgent ear-nose-throat (ENT) referral was made to perform a rigid bronchoscopy to rule out a left main bronchus FB. The ENT consultant advised an urgent CT virtual bronchoscopy (CTVB), which did not reveal any FB (figure 3 ). The parents refused consent for performing a rigid bronchoscopy.

\section{DIFFERENTIAL DIAGNOSIS}

By the time the patient was evaluated by us it was very clear that the diagnosis was not bronchial asthma. Hence discussion on the differential diagnosis of the initial symptoms (recurrent

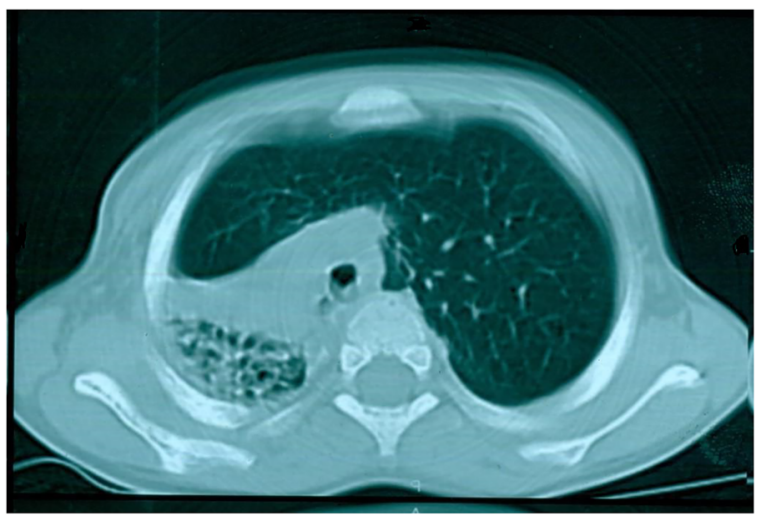

Figure 2 High-resolution chest CT showing collapse of all segments of the left lung with cystic bronchiectatic changes and a massively hyperinflated right lung, resulting in mediastinal shift to the left.

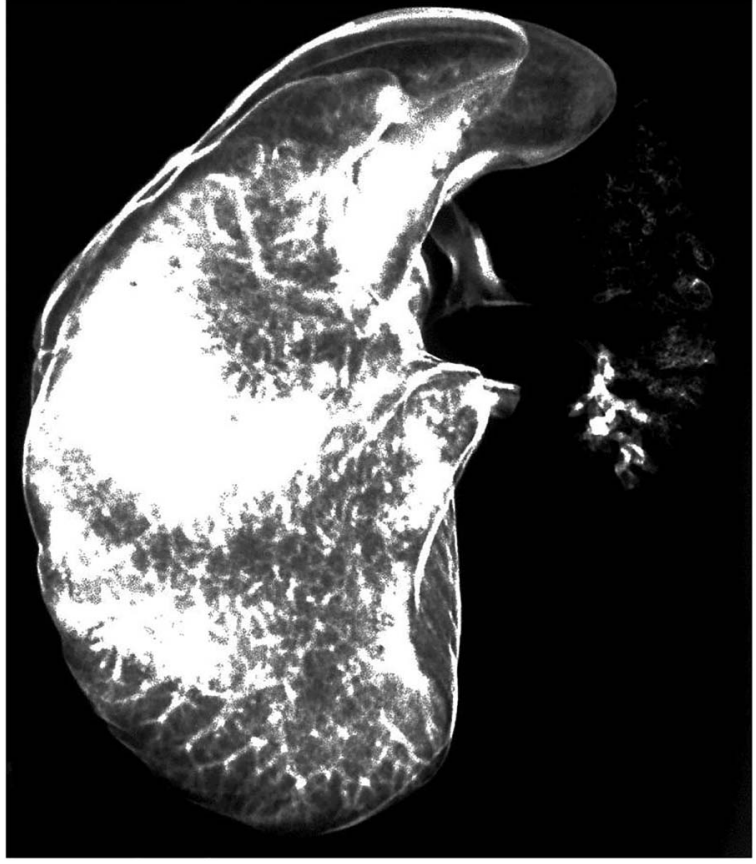

Figure $3 \mathrm{CT}$ virtual bronchoscopy image showing absence of a foreign body.

episodes of cough, wheezing and fever) is not the focus of this report. We wish to discuss the differential diagnoses in the present case at the time of admission to our hospital; namely, when collapse of the entire left lung with diffuse bronchiectasis along with a massive compensatory hyperinflation of the right lung had developed:

1. Chronic FB lodged in the left main bronchus (almost certain)-Total collapse of one lung with compensatory emphysema of the other lung may be due to a chronic FB in its main bronchus unless proven otherwise. ${ }^{1-3} \mathrm{~A} \mathrm{FB}$ in the left main bronchus would have led to its occlusion and resultant total collapse of the left lung. Recurrent superadded pyogenic infections of the collapsed lung would have eventually led to diffuse bronchiectasis.

Chronic FB lodged in the left main bronchus was our first differential diagnosis in spite of there being no history of anyone having witnessed an aspiration episode; and HRCT chest and CTVB not having detected any FB. Timely bronchoscopy in such a scenario would ensure removal of the FB as well as being therapeutic and life-saving. ${ }^{1-3}$

2. Endobronchial tuberculosis (EBTB) of the left main bronchus (possible)-EBTB is a form of pulmonary tuberculosis, thought to be the consequence of rupture of infected tuberculous lymph nodes through the bronchial wall or from lymphatic spread to the mucosal surface of the bronchial tree. $^{5}$ EBTB of the left main bronchus would have led to total collapse of the left lung due to inflammatory secretions and left bronchial stenosis. Recurrent super-added pyogenic infections of the collapsed lung would have eventually led to diffuse bronchiectasis. ${ }^{5}$ Bronchoscopy in such a scenario would detect extrinsic compression by lymph nodes, in addition to an intraluminal mass, ulceration or mucosal inflammation. ${ }^{5}$ Bronchial biopsies and bronchial lavage cultures ('Xpert TB test') for Mycobacterium tuberculosis would have aided in either confirming or ruling out the diagnosis of EBTB. $^{5}$ 
In the present case, HRCT of the chest did not reveal any enlarged caseous lymph nodes compressing the left main bronchus and did not show stenosis of the left main bronchus. However, keeping in mind that tuberculosis is rampant in our country and that it can have protean manifestations, as a matter of caution, we decided to continue antituberculosis treatment in this patient until bronchial lavage culture reports were available to us.

3. Bronchogenic cyst compressing the left main bronchus (unlikely)-Bronchogenic cyst is a rare benign tumour from the primitive foregut and is mostly located in the posterior or middle mediastinum; it can mimic a chronic bronchial FB. ${ }^{6}$ In the present case, a bronchogenic cyst could have been growing insidiously, eventually compressing and occluding the left main bronchus. Resultant total collapse of the left lung would have similarly led to diffuse bronchiectasis.

In the present case, HRCT of the chest did not reveal any cystic lesion growing to compress the left main bronchus, in the mediastinum.

4. A chronic mucus plug in the left main bronchus (most unlikely) - A mucus plug can form in a main bronchus with resultant total lung collapse and diffuse bronchiectasis. ${ }^{7}$ Toddlers who have respiratory muscle weakness and resultant impaired cough reflex (eg, in those having spinal muscular atrophy, congenital myopathies, or spastic quadriparesis) can develop this scenario, especially after an intercurrent lower respiratory tract viral infection. ${ }^{8}$ Our patient's neurological examination was normal.

Progressive collapse and destruction of an entire lung is an uncommon but recognised manifestation of cystic fibrosis. ${ }^{9}$ To date, eight patients, diagnosed as having cystic fibrosis since infancy, have been reported to develop unilateral lung collapse and bronchiectasis with compensatory hyperinflation of the contra-lateral lung during their adult life. ${ }^{9}$ Reasons for development and impaction of thick mucus in the main bronchus in these eight adult cases are unclear; but suboptimal adherence with medication and airway clearance leading to chronic infection with Pseudomonas aeruginosa, increased viscosity of bronchial secretions due to hyperglycaemia and associated gastro-oesophageal reflux disease have been postulated to be the causative predisposing factors. ${ }^{9}$ The present case was a toddler; hence the diagnosis of cystic fibrosis was most unlikely. Additionally, there was no history of meconium ileus or saltytasting skin, and no foul-smelling sticky stools, in the present case.

\section{TREATMENT}

In view of the severe respiratory distress and risk of aspiration, the patient was admitted to the paediatric intensive care unit, kept nil by mouth and started on maintenance intravenous fluids along with intravenous amoxicillin-clavulanic acid (amoxicillin $100 \mathrm{mg} / \mathrm{kg} /$ day + clavulanic acid $20 \mathrm{mg} / \mathrm{kg} /$ day in four divided doses). Simultaneously, the patient was started on nebulisation with standard doses of salbutamol, ipratropium bromide and $\mathrm{N}$-acetyl cysteine. Antituberculosis treatment was continued. Intravenous hydrocortisone was given for 3 days for the severe bronchospasm, with an initial loading dose of $8 \mathrm{mg} / \mathrm{kg}$ followed by $8 \mathrm{mg} / \mathrm{kg} /$ day in four divided doses-after which the respiratory distress settled-and the patient was transferred to the paediatric ward for further management. Subsequently, oral prednisolone $2 \mathrm{mg} / \mathrm{kg} / \mathrm{day}$ in two divided doses was substituted and the patient was orally started on the recommended F-75 diet for SAM. ${ }^{10}$ Within a week of admission, the child became afebrile and the bronchospasm was relieved. Nine days after admission (2 days after the patient's vital parameters had normalised) a trainee paediatrician, during the evening ward rounds, prescribed chest physiotherapy to clear the patient's secretions. Within an hour of the chest physiotherapy (a 15 min session comprising percussion and vibrations, with the patient lying down in right lateral position, for postural drainage) being given, the patient experienced acute respiratory deterioration, started gasping and rapidly deteriorated in the ward. In spite of all resuscitative measures, the patient could not be revived. A complete autopsy was ordered to explain the cause of death.

\section{OUTCOME}

A complete autopsy was performed. The right lung was larger than the left and was hyperinflated. The left lung was small in size with diffuse pleural thickening (figure 4A). The part of the right bronchus just before its bifurcation felt hard on palpation. A longitudinal section of the tracheobronchial tree revealed that this hardness was due to an irregular firm blackish-brown FB $(1.0 \times 0.5 \mathrm{~cm})$, which was covered with mucin and whitish material (figure 4A). The FB was processed for histology and it showed the presence of sclerenchymatous plant cells that were inherently brown (figure 4B) and covered by a suppurative exudate, which contained septate, slender hyphal elements (figure 4C). The morphology of the fungus resembled that of the Aspergillus spp. The FB was a betel nut piece (figure 4A; inset photograph). Apart from the diffuse hyperinflation, the right lung on cut surface did not reveal any abnormality (figure $5 \mathrm{~A}$ ), while the shrunken left lung showed symmetrical, diffuse quiescent bronchiectasis (figure 5B). No fungi were demonstrated in either lung. There was no evidence of tuberculosis. The other organs were normal.

\section{DISCUSSION}

The reasons for the present case being at risk for $\mathrm{FB}$ aspiration were commensurate with those of any other young child aged 1-3 years of age, and included: (1) a tendency to put everything into the mouth and to be easily distracted while eating, (2) inability to chew food properly due to incomplete dentition, (3) immature swallowing coordination and (4) immaturity of laryngeal protective mechanisms. ${ }^{1}$ Of the $\mathrm{FBs}, 66-90 \%$ are organic materials, such as nuts, seeds, fruits and beans, usually leading to fever and pneumonia, as they cause dense local inflammatory response. ${ }^{12}$ If an organic FB (eg, betel nut, as in the present case) is left undetected for more than 30 days, bronchiectatic changes start developing. ${ }^{2}$ It is not uncommon for a child with an undiagnosed bronchial FB to be misdiagnosed in the initial stages as having bronchial asthma. ${ }^{11}$ The present case re-emphasises the caveat that 'all that wheezes is not asthma'.

In late presenting bronchial FBs, a HRCT chest scan may depict alteration of the bronchial wall (indirect evidence of a lodged FB); and, in addition, it will clearly delineate the secondary pulmonary damage. ${ }^{12} 13$ A CTVB is another non-invasive, safe and sensitive diagnostic modality for a bronchial FB. ${ }^{13}{ }^{14}$ In current practice, when a chronic bronchial FB is suspected, performing both, HRCT of the chest and CTVB, prior to rigid bronchoscopy, is recommended practice. ${ }^{12-14}$ CTVB plays an important, complementary diagnostic role as it can help identify the exact location, size, shape and type of FB, and can aid in reducing the time taken for carrying out rigid bronchoscopy. ${ }^{15}$ However, as happened in the present case, HRCT and CTVB may not identify a chronically lodged bronchial FB. The sensitivity for HRCT ranges between $90 \%$ and $100 \%$ while for CTVB it is $80 \% .{ }^{12}$ It is known that the dense inflammatory 
Figure 4 (A) Posterior view of the lungs showing a massively hyperinflated right lung (RL); and a small left lung (LL) with diffuse pleural thickening. Longitudinal section of the tracheobronchial tree showing an irregular firm blackish-brown foreign body $(1.0 \times 0.5 \mathrm{~cm})$ (marked with arrow) in the right bronchus just before its bifurcation. Trachea (T) and left bronchus (LB) were empty. Inset shows close-up of foreign body ('betel nut piece'). (B) Foreign body histology showing presence of inherently brown sclerenchymatous plant cells (Haematoxylin-eosin stain). (C) Suppurative exudate covering foreign body showing septate, slender hyphal elements resembling Aspergillus spp fungus (Gomori methenamine silver stain).

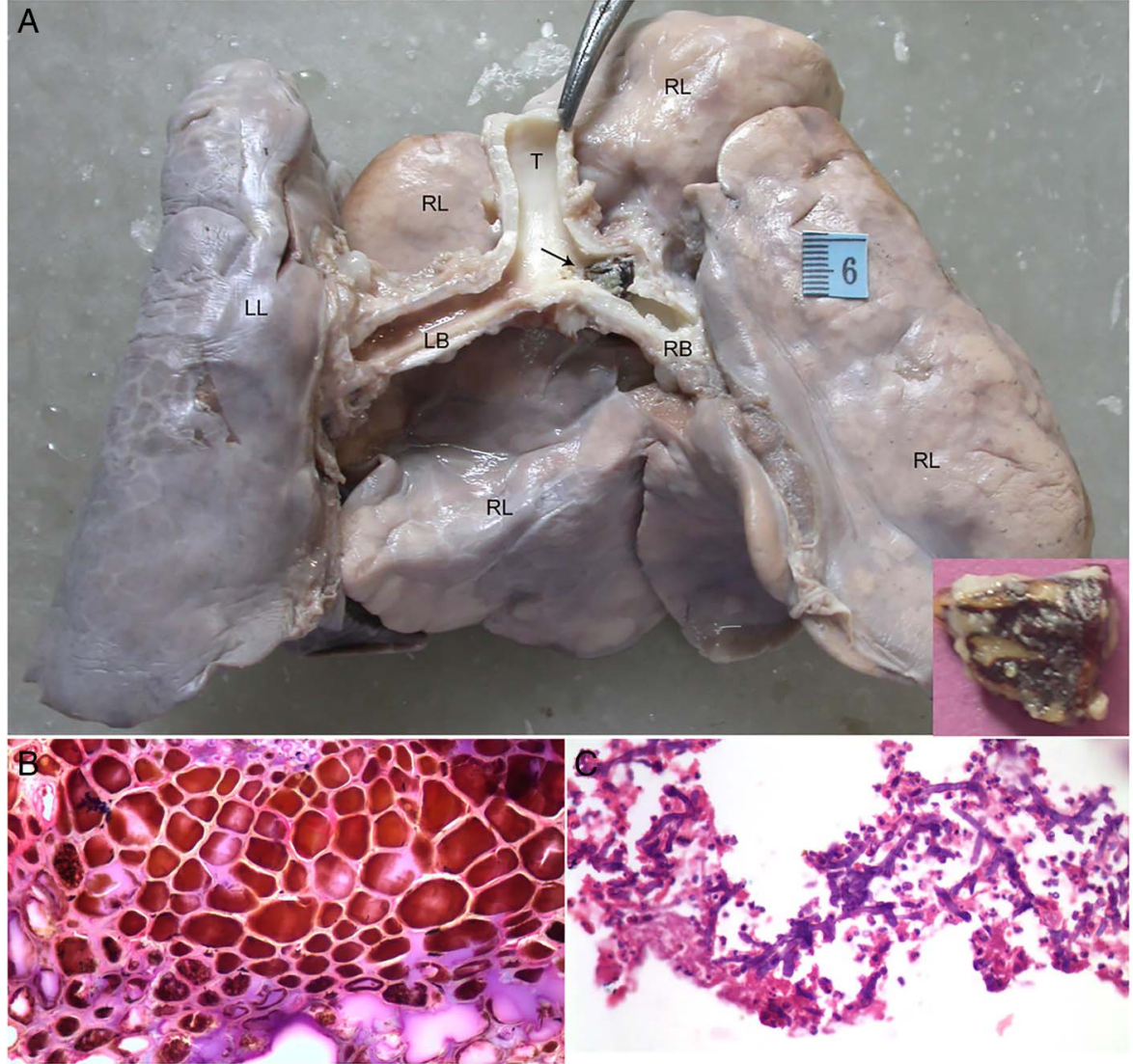

response around a chronic bronchial $\mathrm{FB}$ can obscure the FB from being identified on CT imaging. ${ }^{12}$

Rigid bronchoscopy under general anaesthesia, although an invasive procedure, is an extremely accurate surgical technique to diagnose and remove a FB, and is considered the standard of care in children. ${ }^{17}$ In case of an uncertain diagnosis of a chronic bronchial FB, as in the present case, the decision for performing an explorative rigid bronchoscopy is difficult, as the procedure, although safe in experienced hands, still has the remote risk for serious complications, which can include: severe laryngeal oedema or bronchospasm, requiring tracheotomy or reintubation, pneumothorax, pneumomediastinum, cardiac arrest, tracheal or bronchial laceration and hypoxic brain damage $(0.96 \%){ }^{17}$
Reported mortality during rigid bronchoscopy is $0.42 \% .{ }^{17}$ Heyer et $a l^{18}$ identified three independent predictors of $\mathrm{FB}$, which include: (1) focal hyperinflation on chest radiograph, (2) witnessed choking crisis and (3) white blood cell count greater than $10.0 \times 10^{9} / \mathrm{L}$. They reported that bronchoscopy can be strongly recommended in the presence of at least two risk factors when FB aspiration is suspected. ${ }^{18}$ The present case had two predictors, namely, focal hyperinflation on chest radiograph and white blood cell count greater than $10.0 \times 10^{9} / \mathrm{L}$. Performing a rigid bronchoscopy in the present case was hence indicated, but the patient's parents declined to give informed consent. On hindsight, we wish we had been a bit more persuasive in obtaining parental consent for performing a rigid bronchoscopy.
Figure 5 (A) Cut surface of right lung showing no abnormality apart from the diffuse hyperinflation. (B) Cut surface of shrunken left lung showing symmetrical, diffuse quiescent bronchiectasis.

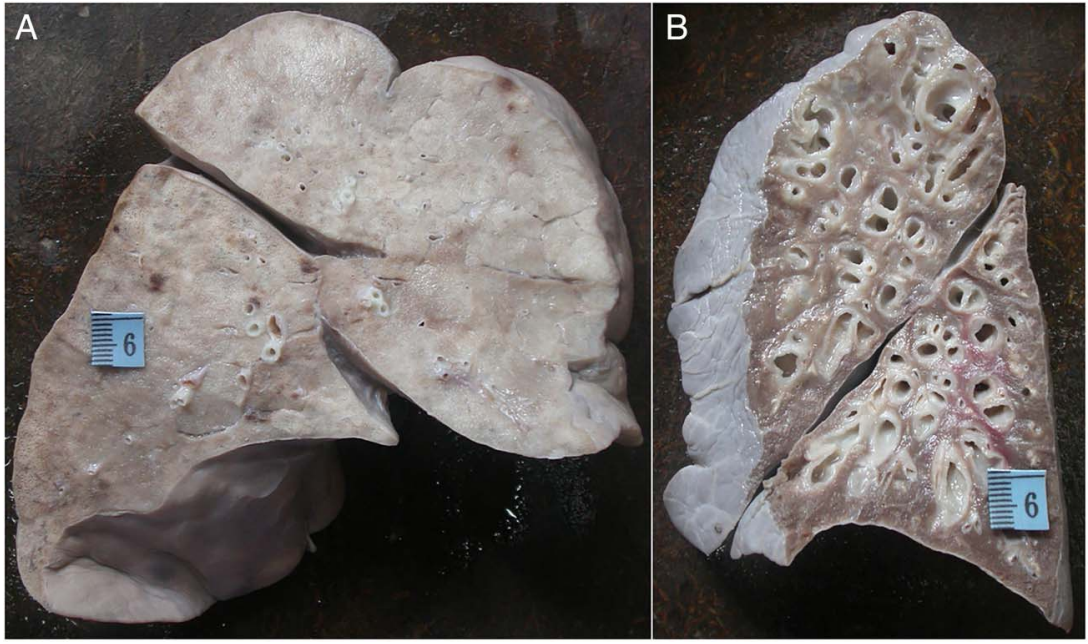

Karande S, et al. BMJ Case Rep 2015. doi:10.1136/bcr-2015-212919 
Ultimately, the autopsy results unravelled the detailed diagnosis and cause of death. The reconstructed sequence of events would be as follows: The betel nut piece had got aspirated and lodged in the left main bronchus after an unwitnessed aspiration episode more than 5 months prior. Over a period of time, the betel nut piece had caused local inflammation and got embedded, and, due to its innate hygroscopic characteristic, swelled up and caused total blockage of the left main bronchus leading to complete collapse of the left lung, with resultant massive compensatory hyperinflation of the right lung. Subsequent repeated pyogenic infections of the collapsed non-functional left lung had led to diffuse bronchiectasis. A young child with SAM is immune-compromised and is known to be at increased risk of developing severe and recurrent lower respiratory tract infections. ${ }^{19}$ Comorbid SAM in our patient, as well, would have contributed to the development of the diffuse bronchiectasis. Following the chest physiotherapy session the FB had got dislodged from its initial site in the left main bronchus and migrated to block the right main bronchus leading to acute respiratory deterioration due to vagal response, devastating airway spasm and eventual death. It is also possible that the salbutamol, ipratropium bromide and $\mathrm{N}$-acetyl cysteine nebulisations along with steroids (hydrocortisone and later prednisolone) therapy, reduced the local inflammation around the embedded FB, relieved the bronchospasm and contributed to the FB getting dislodged following chest physiotherapy.

There have been only three cases reported in the paediatric age group, where an aspirated FB got dislodged and led to acute respiratory deterioration. ${ }^{20-22} \mathrm{Wu}$ and $\mathrm{Wang}^{20}$ reported a case of a 9-year-old boy who had recently choked on a peanut, in whom the FB got dislodged from right to left bronchus after several episodes of stress coughing while awaiting elective rigid bronchoscopy. Sharma et $a l^{21}$ reported a case of a 9-year-old girl who had aspirated a marble 3 months prior, in whom, during right bronchotomy, the FB got dislodged and migrated to block the left bronchus, causing life-threatening hypoxaemia. Graw-Panzer et $a l^{22}$ reported a case of a 27-month-old baby in whom a previously undetected FB ('a plastic piece') got dislodged from the right main and shifted to the left main bronchus while the patient was intubated and mechanically ventilated, leading to acute respiratory deterioration. This patient had been admitted for status epilepticus and on admission was detected to have a pneumonia that progressed to respiratory failure and acute respiratory distress syndrome.

Learning points

- Persistent cough, recurrent wheezing/pneumonia and fever are red flag symptoms of a long-standing bronchial foreign body (FB).

- The most frequent finding of a chronic bronchial FB on chest radiograph is atelectasis with diffuse bronchiectasis.

- Total collapse of one lung with compensatory emphysema of the other lung is due to a FB in its main bronchus unless proven otherwise.

- It is possible to miss a radiolucent chronic bronchial FB on high-resolution CT chest as well as on CT virtual bronchoscopy.

- Chest physiotherapy can dislodge a chronic bronchial FB, with tragic consequences.
Agitation of the patient, coughing and airway clearance manoeuvres, such as chest percussion and positional drainage, led to dislodgment of the FB in this intubated patient. ${ }^{22}$ Fortunately, all three patients in the cases mentioned above survived with appropriate emergency management. ${ }^{20-22}$

The present case highlights the fact that a chronic embedded bronchial FB can get dislodged after chest physiotherapy, with tragic consequences. This is the lesson we learnt from our error. In future, for cases of suspected FB aspiration, we will be prominently displaying the point that 'chest physiotherapy is NOT to be given', in the patient's treatment notes. It is not uncommon for unindicated chest physiotherapy to be prescribed inadvertently by overworked trainee doctors. ${ }^{23}$ Lastly, the present case highlights the ills of betel nut consumption and the need for parental and family education to reduce the incidence of this preventable complication.

Acknowledgements The authors thank our Dean, Dr AN Supe, for granting us permission to publish this case report.

Contributors SK and MM were involved in the clinical management of the patient. PV performed the autopsy and interpreted its findings. SK, PV and MM were involved in writing the manuscript and reviewing the literature, and have seen and approved the final version of the manuscript.

Competing interests None declared.

Patient consent Not obtained.

Provenance and peer review Not commissioned; externally peer reviewed.

\section{REFERENCES}

1 Tomaske M, Gerber AC, Stocker $\mathrm{S}$, et al. Tracheobronchial foreign body aspiration in children-diagnostic value of symptoms and signs. Swiss Med Wkly 2006;136:533-8.

2 Karakoc F, Karadag B, Akbenlioglu C, et al. Foreign body aspiration: what is the outcome? Pediatr Pulmonol 2002;34:30-6.

3 Boufersaoui $\mathrm{A}$, Smati L, Benhalla $\mathrm{KN}$, et al. Foreign body aspiration in children: experience from 2624 patients. Int J Pediatr Otorhinolaryngol 2013;77:1683-8.

4 WHO, UNICEF. WHO child growth standards and the identification of severe acute malnutrition in infants and children. A Joint Statement by the World Health Organization and the United Nations Children's Fund. Geneva: World Health Organization and United Nations Children's Fund, 2009.

5 Kashyap S, Mohapatra PR, Saini V. Endobronchial tuberculosis. Indian J Chest Dis Allied Sci 2003:45:247-56.

6 Mampilly T, Kurian R, Shenai A. Bronchogenic cyst-cause of refractory wheezing in infancy. Indian J Pediatr 2005;72:363-4.

7 Raman TS, Mathew S, Ravikumar, et al. Atelectasis in children. Indian Pediatr 1998:35:429-35.

8 Finder JD. Airway clearance modalities in neuromuscular disease. Paediatr Respir Rev 2010;11:31-4.

9 Flight WG, Hildage J, Kevin Webb A. Progressive unilateral lung collapse in cystic fibrosis - a therapeutic challenge. J R Soc Med 2012;105(Suppl 2):S44-9.

10 WHO. Guideline: updates on the management of severe acute malnutrition in infants and children. Geneva: World Health Organization, 2013.

11 Maguire A, Gopalakaje S, Eastham K. All that wheezes is not asthma: a 6-year-old with foreign body aspiration and no suggestive history. BMJ Case Rep 2012;2012: pii: bcr2012006640.

12 Tuckett $\mathrm{P}$, Cervin A. Reducing the number of rigid bronchoscopies performed in suspected foreign body aspiration cases via the use of chest computed tomography: is it safe? A literature review. J Laryngol Otol 2015;129(Suppl 1):S1-7.

13 Adaletli I, Kurugoglu S, Ulus $\mathrm{S}$, et al. Utilization of low-dose multidetector CT and virtual bronchoscopy in children with suspected foreign body aspiration. Pediatr Radiol 2007;37:33-40

14 Jung SY, Pae SY, Chung SM, et al. Three-dimensional CT with virtual bronchoscopy: a useful modality for bronchial foreign bodies in pediatric patients. Eur Arch Otorhinolaryngol 2012;269:223-8.

15 Bhat KV, Hegde JS, Nagalotimath US, et al. Evaluation of computed tomography virtual bronchoscopy in paediatric tracheobronchial foreign body aspiration. J Laryngol Otol 2010;124:875-9.

16 Halwai O, Bihani A, Dabholkar J. A study of clinical presentations and complications of foreign body in the bronchus-our experience. Otolaryngol Pol 2015;69:22-9.

17 Fidkowski CW, Zheng $\mathrm{H}$, Firth PG. The anesthetic considerations of tracheobronchial foreign bodies in children: a literature review of 12,979 cases. Anesth Analg 2010;111:1016-25 


\section{Learning from errors}

18 Heyer CM, Bollmeier ME, Rossler L, et al. Evaluation of clinical, radiologic, and laboratory prebronchoscopy findings in children with suspected foreign body aspiration. J Pediatr Surg 2006;41:1882-8.

19 Jones KD, Berkley JA. Severe acute malnutrition and infection. Paediatr Int Child Health 2014;34(Suppl 1):S1-29.

20 Wu CT, Wang CJ. Alternate lung collapse in a 9-year-old boy with peanut aspiration. Pediatr Radiol 2006;36:1327.
21 Sharma $P$, Kumar $A$, Kumar $A$. Intraoperative airway foreign body migration in a child. Anaesth Intensive Care 2009;37:1021-4.

22 Graw-Panzer KD, Wadowski SJ, Lee H. Complicated and dislodged airway foreign body in an intubated child: case report. Pediatr Emerg Care 2012;28:915-17.

23 Harding S, Smith ME, Watson A. Chest physiotherapy. BMJ 1989; 298:826-7.

Copyright 2015 BMJ Publishing Group. All rights reserved. For permission to reuse any of this content visit http://group.bmj.com/group/rights-licensing/permissions.

BMJ Case Report Fellows may re-use this article for personal use and teaching without any further permission.

Become a Fellow of BMJ Case Reports today and you can:

- Submit as many cases as you like

- Enjoy fast sympathetic peer review and rapid publication of accepted articles

- Access all the published articles

- Re-use any of the published material for personal use and teaching without further permission

For information on Institutional Fellowships contact consortiasales@bmjgroup.com

Visit casereports.bmj.com for more articles like this and to become a Fellow 